\title{
The Entire Trajectories of Single Olivocerebellar Axons in the Cerebellar Cortex and their Contribution to Cerebellar Compartmentalization
}

\author{
I. Sugihara, ${ }^{1}$ H.-S. Wu, ${ }^{1,2}$ and Y. Shinoda ${ }^{1,2}$ \\ ${ }^{1}$ Department of Systems Neurophysiology, Tokyo Medical and Dental University Graduate School of Medicine, \\ Bunkyo-ku, Tokyo 113-8519, Japan, and 2The Core Research for Evolutional Science and Technology Program, \\ Kawaguchi, 332-0012, Japan
}

The functional partitioning of the cerebellar cortex depends on the projection patterns of its afferent and efferent neurons. However, the entire morphology of individual projection neurons has been demonstrated in only a few classes of neurons in the vertebrate CNS. To investigate the contribution of the projection pattern of individual olivocerebellar axons to the cerebellar functional compartmentalization, we labeled individual olivocerebellar axons, which terminate in the cerebellar cortex as climbing fibers, with biotinylated dextran amine injected into the inferior olive in the rat, and completely reconstructed the entire trajectories of 34 olivocerebellar axons from serial sections of the cerebellum and medulla. Single axons had seven climbing fibers on average, which terminated at similar distances from the midline in a single or in multiple lobules. Cortical projection areas of adjacent olivary neurons were clustered as narrow but separate longitudinal segments and often innervated by collaterals of single neurons. Comparison of the cerebellar distribution of olivocerebellar axons arising from different sites within a single olivary subnucleus indicated that slightly distant neurons projected to complementary sets of such segments in a single longitudinal band. Several of these longitudinal bands formed a so-called parasagittal zone innervated by a subnucleus of the inferior olive. Single olivocerebellar axons projected rostrocaudally to segments within a single band but did not project mediolaterally to multiple bands. These results suggest fine substructural organization in the cerebellar compartmentalization that may represent functional units.

Key words: climbing fibers; biotinylated dextran amine; inferior olive; rats; cerebellar cortex; cerebellum; afferent neurons; neural pathways; neuroanatomy; brain mapping
The uniformity of the local circuit of the cerebellar cortex (Ramón y Cajal, 1911; Eccles et al., 1967; Palay and Chan-Palay, 1974; Ito, 1984) implies that functional partitioning of the cerebellar cortex depends on the patterns of afferent and efferent connections to each cerebellar cortical region. Indeed, anatomical studies have shown that the mammalian cerebellar cortex can be divided into several parasagittal zonal compartments (designated A, B, C1-3, and D1-2) on the basis of the topography of the olivocerebellar projection (Groenewegen and Voogd, 1977; Azizi and Woodward, 1987; Buisseret-Delmas and Angaut, 1993; Voogd, 1995). However, electrophysiological studies involving sensory mapping have suggested an even finer compartmentalization in which the A-D zones are subdivided into narrower "microzones" (Oscarsson and Sjölund, 1977; Ito, 1984; Ekerot et al., 1991; Jörntell et al., 2000). Multiple electrode and optical recording studies have also identified such narrow longitudinal microzones in the cerebellar cortex based on correlations in Purkinje cell complex spike activity (Llinás and Sasaki, 1989; Hansen et al.,

\footnotetext{
Received May 14, 2001; revised July 2, 2001; accepted July 9, 2001.

This work was supported by Core Research for Evolutional Science and Technology of Japan Science and Technology Corporation (Y.S.) and Grants-in-Aid for Scientific Research from the Ministry for Education, Science, and Culture of Japan (I. S., Y. S.). We thank Dr. E. J. Lang for reading this manuscript and Dr. K. Miura of FujiFilm for his support of the computer-aided dynamic focusing system.

Correspondence should be addressed to Dr. Yoshikazu Shinoda, Department of Systems Neurophysiology, Tokyo Medical and Dental University, Graduate School of Medicine, 1-5-45 Yushima, Bunkyo-ku, Tokyo 113-8519 Japan. E-mail: yshinoda.phy1@tmd.ac.jp.

Copyright (C) 2001 Society for Neuroscience $\quad 0270-6474 / 01 / 217715-09 \$ 15.00 / 0$
}

2000). Thus, the olivocerebellar projection appears to be organized into many more functional zones than have been identified anatomically. Several fine zones have been anatomically demonstrated in the flocculus and nodule (Ruigrok et al., 1992; Tan et al., 1995); however, in general, morphological correlates for electrophysiologically identified narrow zones in the olivocerebellar projection remain elusive.

One way to attempt to identify a finer anatomical organization to the olivocerebellar system is to analyze the projection patterns of individual olivary axons. Recently, the complete trajectories of single axons of inferior olive neurons have been reconstructed (Sugihara et al., 1999). Individual olivary neurons project to rather localized areas with several climbing fibers by ramifying mostly in the cerebellar white matter and occasionally in the cerebellar granular layer (Sugihara et al., 1999). This conforms to the idea that the projection pattern of olivocerebellar axons is tightly related to a compartmentalization of the cerebellar cortex and suggests that the analysis of individual axonal projections is useful for understanding the morphological correlates underlying the functional compartmentalization of the cerebellar cortex. Thus, the present study was undertaken to investigate the basic structural organization of the olivocerebellar projection by analyzing the entire axonal trajectories of individual olivary neurons.

\section{MATERIALS AND METHODS}

Tracer injection and histological procedure. Thirty-six adult Long-Evans rats were used. Methods of anesthesia, surgery, and histological procedures and axonal reconstruction were the same as described previously 
(Sugihara et al., 1999). The experimental animals were treated ethically, and the experimental protocols were approved by the Institutional Animal Care and Use Committee in Tokyo Medical and Dental University. Biotinylated dextran amine was pressure-injected into the inferior olive $(0.1$ to $<0.01 \mu \mathrm{l}$ of $10 \%$ solution in saline). The rats in the present study had a small injection with a spread of dye in the injection site limited to $<0.2 \mathrm{~mm}$, unless otherwise stated. Fifty-micrometer-thick serial frozen sections were cut from the entire cerebellum and medulla parasagittally in most cases and in others coronally. The protocols for histological labeling of biotinylated dextran amine were the same as described previously (Sugihara et al., 1999). Some photomicrographs were obtained with a computer-aided dynamic focusing system (MCID image analysis system; Imaging Research, St. Catharines, Ontario, Canada).

Reconstruction of individual axons. Axonal trajectories of single labeled olivocerebellar axons were reconstructed from serial parasagittal sections using a three-dimensional imaging microscope (Edge R400; SNT Microscopes, Los Angeles, CA) equipped with a camera lucida apparatus with objectives of 10, 20, 40, 60 and $100 \times$. Cut ends of an axon on one section were connected properly to the corresponding cut ends of the same axon on the successive section (Shinoda et al., 1981; Sugihara et al., 1999). Only axons that were well labeled, isolated from other axons, and could be traced from the injection site to every climbing fiber terminal were regarded as completely reconstructed, whereas axons that could not be traced at any point on their pathway because of poor labeling or intermingling with other axons were regarded as "not fully reconstructed."

Mapping climbing fiber locations onto the unfolded cerebellar cortex. In the diagram of the unfolded cerebellar cortex, as in Figures $3 b, 5$, and 6 , the distances from the midline to each climbing fiber and the cerebellar outline were to scale (measured by the number of the parasagittal sections). A small difference in the lateral dimension of the cerebellum between animals $(<10 \%)$ was adjusted in the figures by normalizing the maximum width measured at Crus $\mathrm{Ib}$ to the average value $(11.4 \mathrm{~mm})$ obtained from seven rats in which the sections were cut coronally. The rostrocaudal dimension of each cerebellar lobule was determined by measuring the length of the Purkinje cell layer in each lobule in parasagittal sections at the midline and 1.5 and $3 \mathrm{~mm}$ lateral from the midline. The primary fissure was made straight arbitrarily. The rostrocaudal distances in the paraflocculus and flocculus are not to scale (enlarged for clarity). Broken outlines in the paraflocculus and flocculus indicate the continuation of the Purkinje cell layer. When mapping from parasagittal sections, the position in the rostrocaudal axis for each climbing fiber was determined by measuring its relative distance from the borders of the lobule in a parasagittal section, and the mediolateral distance was determined from the number of the sections. When mapping from coronal sections, the parasagittal outline of the tissue at several mediolateral distances was reconstructed first. Climbing fibers were mapped onto these parasagittal outlines and then transferred onto the unfolded cerebellar cortex diagram.

To map climbing fibers in the unfolded hemisphere along a tilted parasagittal plane, as in Figure $4 b$, the three-dimensional coordinates of each climbing fiber were measured at the point where it contacted a target Purkinje cell. The equation for the plane was calculated by minimizing the sum of the squares of the distance from the plane to the individual points. The distance from this plane for each point was used as the mediolateral position in the unfolded hemisphere. A homemade computer program was used for these calculations. The rostrocaudal position of each climbing fiber was measured along the Purkinje cell layer on this plane.

The diagram of the inferior olive represents a dorsal view that was reconstructed from the preparation in which the majority of olivary neurons were labeled retrogradely by a large injection of biotinylated dextran amine into the inferior cerebellar peduncle.

\section{RESULTS}

\section{Branches of individual olivocerebellar axons are confined to a single rostrocaudally oriented plane}

Olivocerebellar axons were labeled with biotinylated dextran (Sugihara et al., 1999), an anterograde neuronal tracer. Injections were made into the inferior olive (Fig. 1c), and the complete trajectories of 34 axons were reconstructed from parasagittal or coronal serial sections in 14 rats. Individual olivocerebellar axons ramified many times, mainly within the deep cerebellar white matter, into thick branches that terminated as climbing fibers in the cerebellar cortex and thin collaterals that terminated mainly in the cerebellar nuclei and the granular layer (Fig. 1a) (Sugihara et al., 1999). The number of climbing fibers per axon ranged from 2 to $17(6.6 \pm 3.7$; mean $\pm \mathrm{SD} ; n=34)$. This number was consistent with the average number of climbing fibers per axon (approximately seven) inferred by counting the total numbers of Purkinje cells and olivary neurons in the rat (Schild, 1970), supporting complete labeling of reconstructed axons. Axons could be classified into two types based on their climbing fiber distribution pattern. For most axons ( $n=22$ of 34) all of their climbing fibers terminated within a single rostrocaudally oriented area that was limited to one lobule or multiple contiguous lobules (Fig. $1 b)$. However, a significant percentage of axons $(n=12$ of 34) had a distinct termination pattern in which climbing fibers were distributed to multiple non-contiguous lobules (Fig. 2). In both cases, all climbing fibers originating from a single neuron were located at almost equal distances from the midline and therefore were contained within a single parasagittal plane (Fig. $1 d)$. The width of these planes was measured as the maximum mediolateral spread of climbing fibers from an individual olivocerebellar axon (the distance between the nearest and farthest climbing fibers from the midline). For reconstructed axons in the vermis the average width was $217 \pm 173 \mu \mathrm{m}(n=16)$. The maximum mediolateral spread of branches was larger (1114 \pm $441 \mu \mathrm{m} ; n=18$ ) for axons terminating in the pars intermedia and hemisphere; however, this was because the plane that contained climbing fibers of individual axons was tilted from the parasagittal plane (Fig. 2b,c). When measured perpendicularly to this tilted plane, the widths of the planes that contained the branches of individual axons were as narrow as in the vermis $(0.2-0.3 \mathrm{~mm})$.

\section{Distribution of axonal branches from neighboring cells}

The activity of adjacent olivary neurons is functionally correlated because of the electrotonic coupling among olivary neurons (Llinás et al., 1974) and because of focal afferent projection patterns to the inferior olive (Apps, 1998; McCurdy et al., 1998). To determine the translation of such correlated olivary activities into patterns of activity across the cerebellar cortex, it is necessary to know the relationship of axonal projection patterns of adjacent olivary neurons. To address this issue, we reconstructed all labeled axons from neighboring olivary cells in each animal. Figure $3 a$ shows an example in which six individual olivocerebellar axons were completely reconstructed from a single injection site. Fortytwo climbing fibers originating from these six axons (Fig. 3a, colored fibers) and 36 climbing fibers originating from other axons (not fully reconstructed, gray fibers) were all located in vermal lobules VIa-c and VII. Within this region there was significant overlap of the projection areas, with individual axons projecting to lobules V Ia, V Ib, and VII (light blue), to lobules V Ia, VIb, and VIc (violet), to lobules VIa and VIb (green), to lobules VIb and VIc (orange and magenta), and to lobule VII (brown). These results indicated that olivary neurons located close to each other shared projection areas. To clarify the mediolateral and rostrocaudal extents of the cortical projection areas shared by adjacent neurons, henceforth referred to as "segments," the labeled climbing fibers shown in Figure $3 a$ were mapped onto an unfolded representation of the cerebellar cortex (Fig. 3b). All labeled climbing fibers were distributed as clustered segments within a narrow rostrocaudally oriented area, which was almost in parallel with the midline. These segments were more or less continuous in a rostrocaudal direction and formed a clearly delineated narrow longitudinal band. The mediolateral width of this longitudinal 

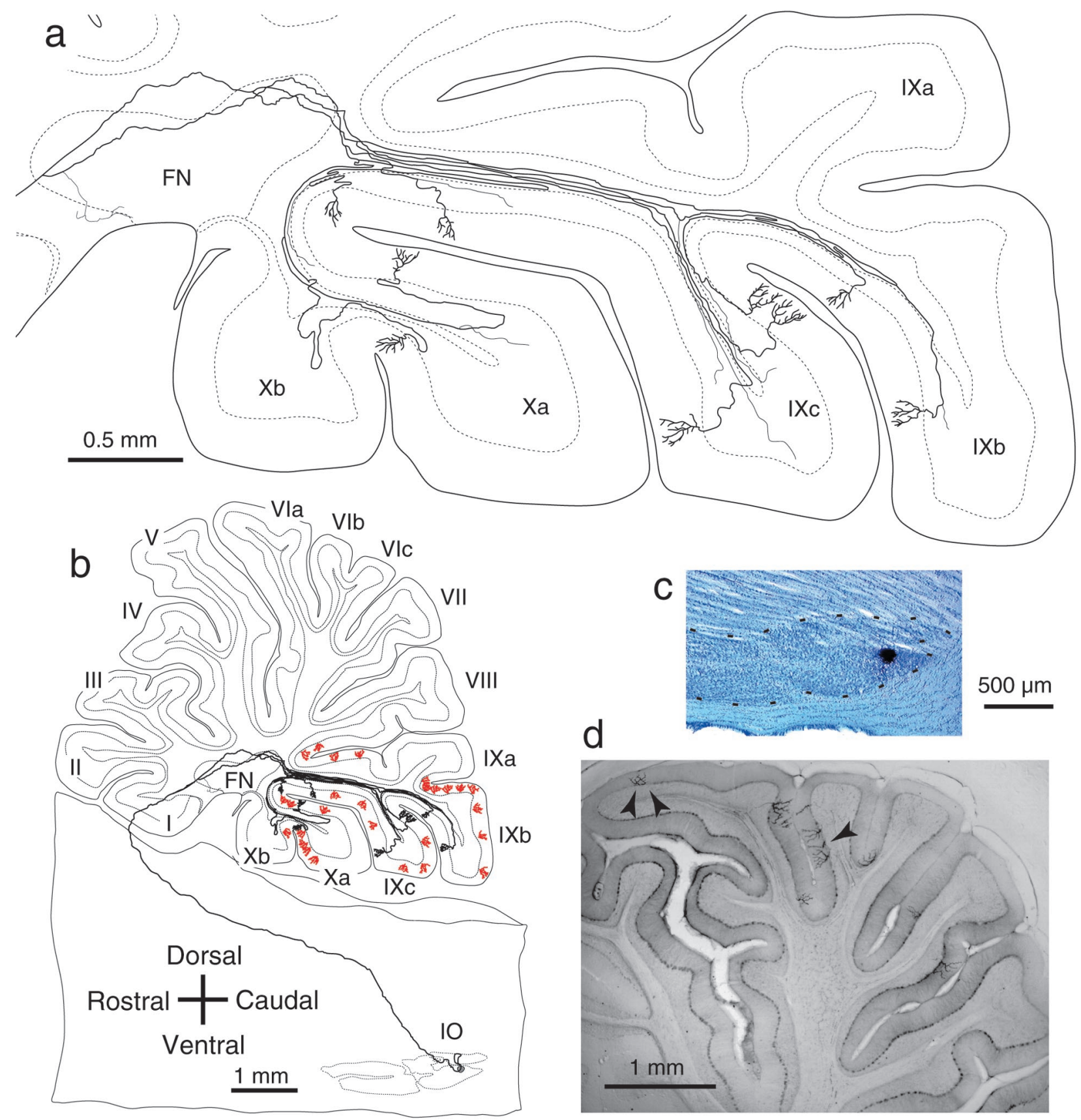

Figure 1. Projection patterns of single olivary axons in the cerebellum. $a$, Intracerebellar trajectory of a single olivocerebellar axon labeled after a small injection of biotinylated dextran amine (BDA) into the mediocaudal portion of the MAO (reconstructed from 77 serial parasagittal sections). This axon gave rise to nine climbing fibers terminating in uvula (lobule IXb-c) and nodule (lobule Xa), and thin collaterals terminating in the cerebellar nucleus and the granular layer. $b$, Entire trajectory of the same axon shown in $a$ with all the other climbing fibers $(n=28$, red $)$ labeled by the tracer injection. All climbing fibers were distributed from caudal lobule VIII to lobule X. $c$, Photomicrograph of the injection site in the inferior olive shown in $b$. $d$, Photomicrograph of labeled climbing fibers in a parasagittal section. Arrowheads indicate the three labeled climbing fibers belonging to a single inferior olive neuron. Digital focusing and enhancement were used. Abbreviations in this and subsequent figures and legends: $I-X$, lobules I-X; $a-d$, sublobules a-d; $C P$, copula pyramidis; $C r I$, crus I ansiform lobule; $C r I I$, crus II ansiform lobule; $D N$, dentate nucleus; $D P F L$, dorsal paraflocculus; $F L$, flocculus; $F N$, fastigial nucleus; $I N$, interposed nucleus; $I O$, inferior olive; $M A O$, medial accessory olive; Par, paramedian lobule; $P O-D L$, dorsal lamella of the principal olive; Sim, simple lobule; VPFL, ventral paraflocculus.

band was $\sim 0.2-0.4 \mathrm{~mm}$ when a slightly curved boundary was fitted (broken line) and was only 3.5-7\% of the entire width of the left vermis. In contrast, the rostrocaudal dimension of this band was almost 40 times as long as the mediolateral dimension.

To examine further whether this narrow longitudinal band structure exists in the cerebellar cortex outside the vermis, a similar analysis was made of axons terminating in the hemisphere (Fig. 4). Axonal trajectories of three well labeled axons were completely reconstructed from a small injection in the principal olive. Climbing fibers originating from these axons (colored) and five other labeled climbing fibers (gray) were located in crus Ia and Ib (Fig. 4a). All climbing fibers were mostly positioned in a 
a

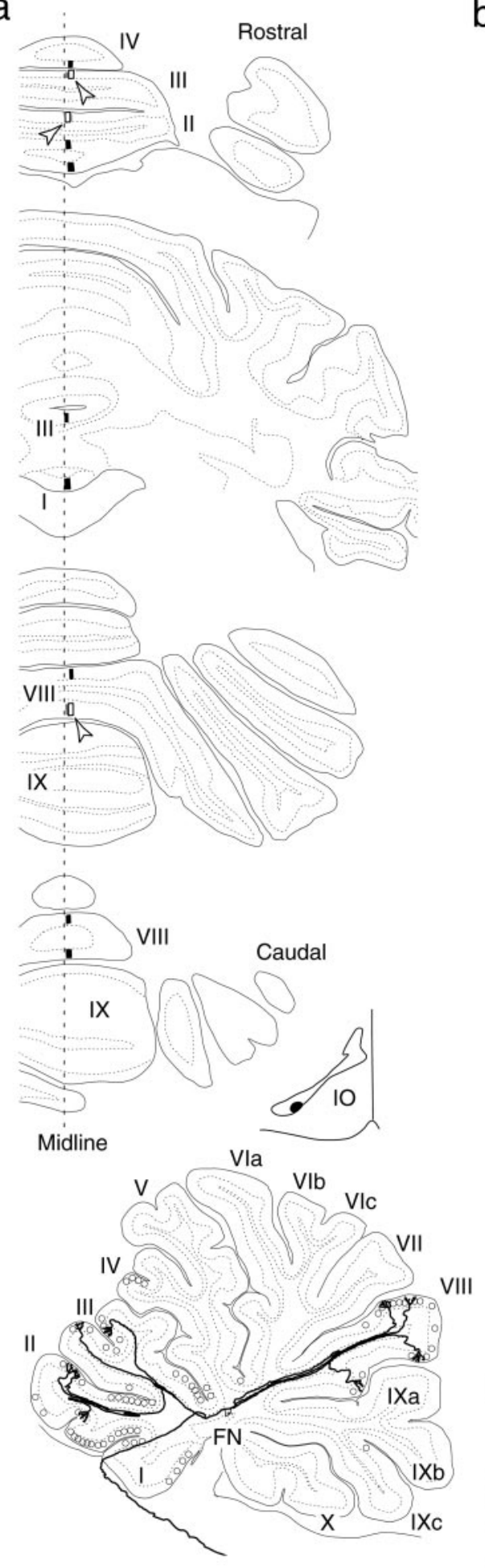

b

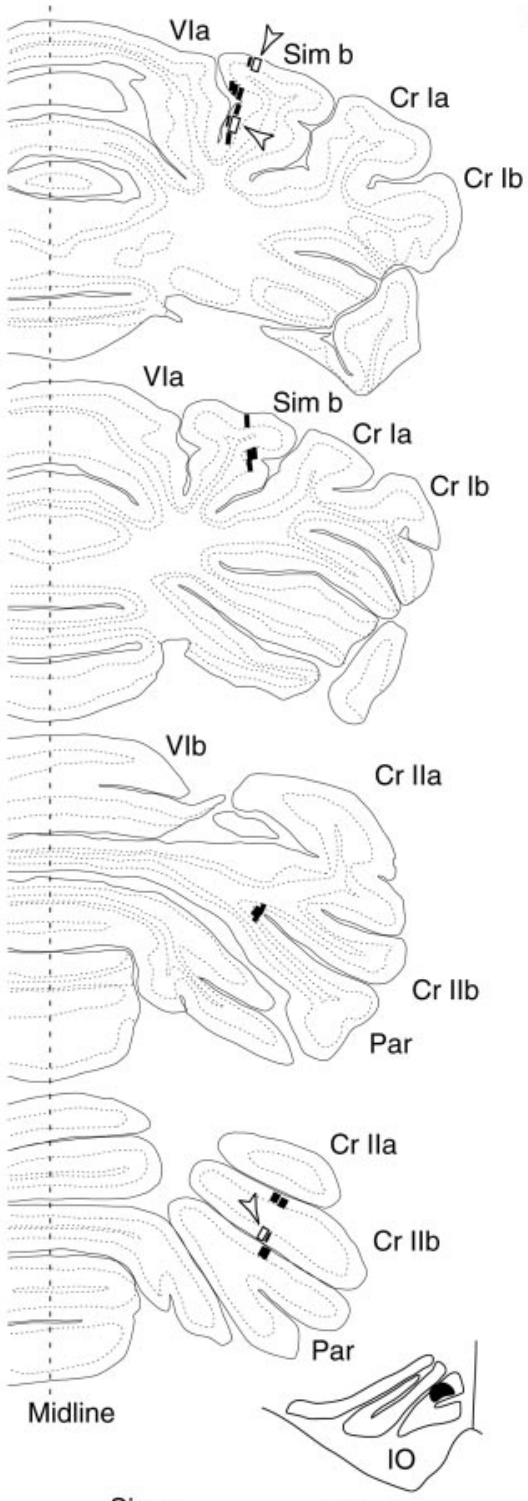

Sim a
C
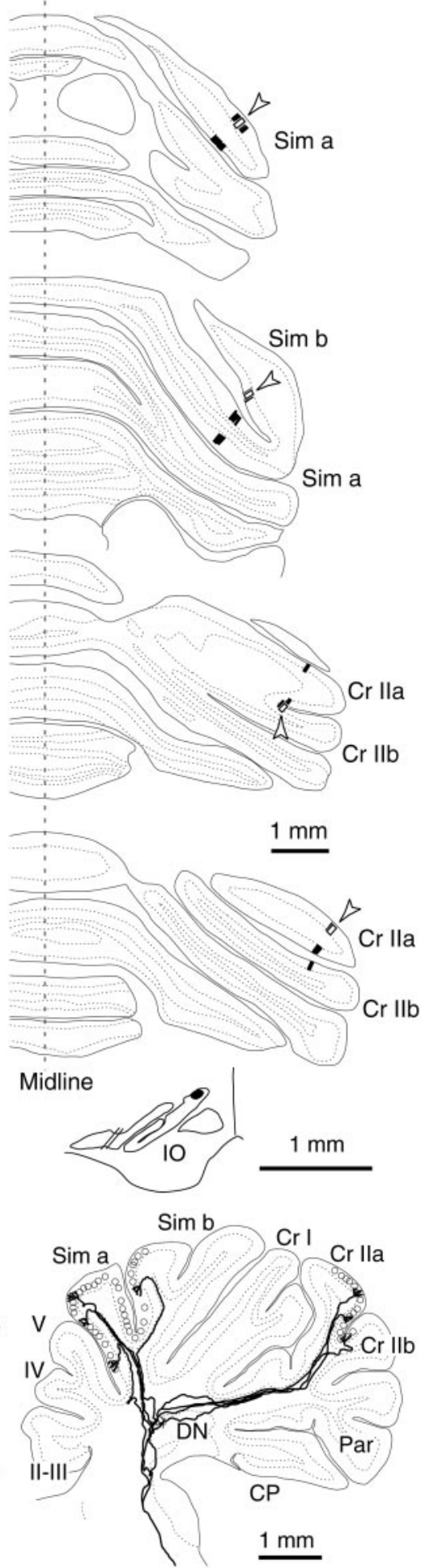

Figure 2. Narrow longitudinal band distribution of climbing fibers originating from single olivary neurons. Results in three animals are shown in individual columns $a-c$, in which climbing fibers were labeled in the vermis $(a)$, pars intermedia $(b)$, and hemisphere $(c)$ by a small tracer injection in the caudal MAO $(a)$, rostral MAO $(b)$, and dorsomedial subnucleus of the ventral leaf of the principal olive $(c)$, respectively. Coronal sections in each column show climbing fibers of a single reconstructed olivary axon (open rectangles indicated by open arrowheads) and other labeled climbing fibers (black bars) that were observed in neighboring four to six sections. A maximum mediolateral spread of labeled climbing fibers was $\sim 0.3 \mathrm{~mm}$. Injection sites are indicated in coronal sections of the inferior olive. The bottom drawing shows a lateral view of a trajectory of reconstructed olivary axon with other labeled climbing fibers (open circles). The climbing fiber distribution areas in $a-c$ belonged to A, C2, and D0 zones (Buisseret-Delmas and Angaut, 1993; Voogd, 1995), respectively. 


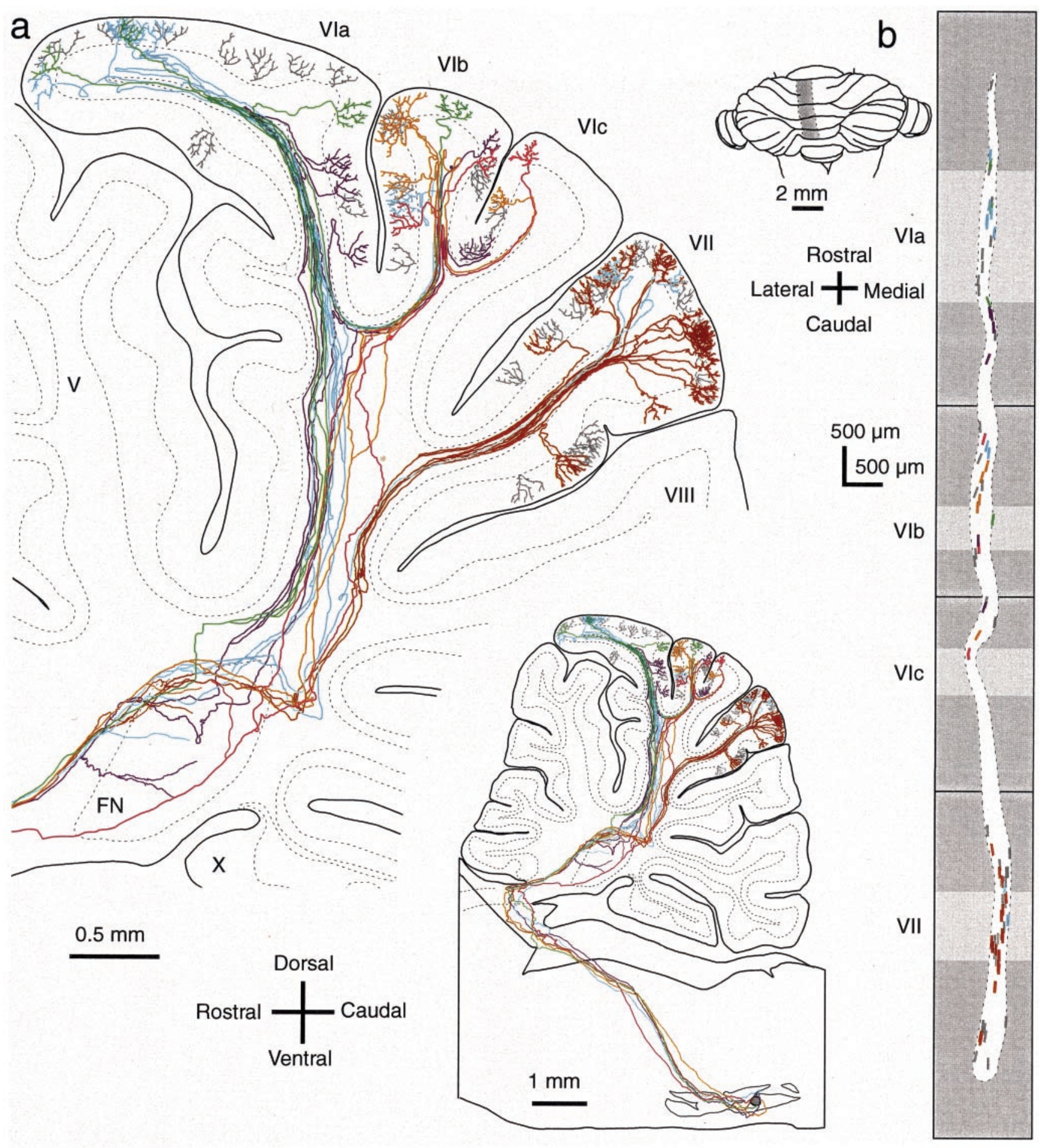

Figure 3. Climbing fibers originating from small areas in the inferior olive distribute within narrow longitudinal bands in the cerebellar cortex. $a$, Lateral view of six olivocerebellar axons (colored) reconstructed from 81 serial parasagittal sections. Forty-two climbing fibers arising from these six axons, and the other 36 labeled climbing fibers ( gray) are included. Inset shows lateral view under low magnification of the entire axonal trajectories from the injection site in the centromedial portion of the MAO (single injection of $0.01 \mu \mathrm{l}$ of BDA). $b$, The distribution of climbing fibers plotted on the unfolded vermal cortex from the midline to the left by $1.3 \mathrm{~mm}$. Colors used for the climbing fibers in $b$ correspond to those used for individual axons in $a$. Light and dark gray areas in the unfolded scheme represent the cerebellar cortex exposed in the cerebellar surface and hidden in the sulci, respectively. Dotted line indicates the contour of the distribution area. Inset shows the area for the unfolded display.

single plane that was tilted laterally (Fig. $4 b$, inset). This tilted plane was numerically obtained (see Materials and Methods), and the cerebellar cortex in the hemisphere was unfolded along this plane. The plot of climbing fibers on the unfolded cortex shows that they were arranged in a single narrow longitudinal band (Fig. $4 b$ ). Although this band was slightly twisted presumably along the foliation of the lobules, the maximum width of the band was 0.25 $\mathrm{mm}$, similar to the width of a band in the vermis. A similar 

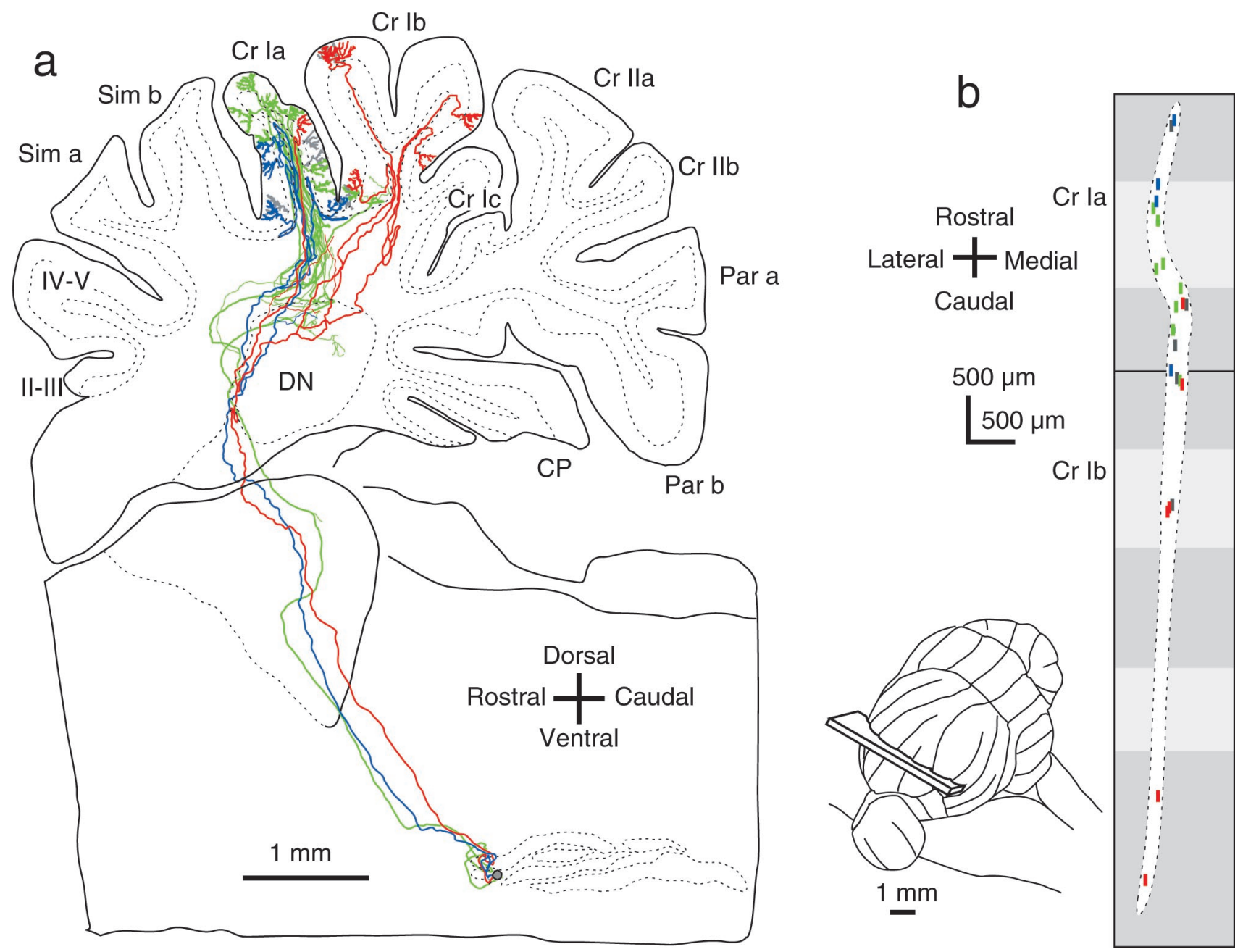

Figure 4. Climbing fiber distribution in a narrow longitudinal area in the hemisphere. $a$, Lateral view of trajectories of three olivocerebellar axons (colored) reconstructed on 31 serial coronal sections. A tracer injection was made in the rostral portion of the dorsal lamella of the principal subnucleus of the inferior olive. Of 23 labeled climbing fibers, 18 climbing fibers arose from these three axons, and the other five labeled climbing fibers ( gray) from other olivary neurons. The outline of the cerebellum is deformed to show all climbing fibers. $b$, The distribution of climbing fibers was plotted on the unfolded hemisphere along a tilted parasagittal plane. Inset shows the tilted parasagittal plane in which climbing fibers were aligned. Colors used for the climbing fibers in $b$ correspond to those used for individual axons in $a$. Light and dark gray areas in the unfolded scheme represent the cerebellar cortex exposed in the cerebellar surface and hidden in the sulci, respectively. Dotted line indicates the contour of the distribution area.

longitudinal band with extensive overlap of axonal projection areas of neighboring neurons in the dorsal cap of the inferior olive was observed in the flocculus (six axons) in another rat.

To obtain a general view of the olivocerebellar organization in the entire cerebellar cortex, tracer injections were made into small areas within the various subnuclei of the inferior olive in 30 rats; the caudal portion of the medial accessory olive (MAO) ( $n=12$ animals), the rostral portion of the MAO $(n=4)$, the dorsal accessory olive (DAO) $(n=6)$, the principal olive (PO) $(n=6)$, and the dorsal cap $(n=2)$. These injections resulted in labeled climbing fibers in the vermis, pars intermedia, pars intermedia, hemisphere, and flocculus, respectively, consistent with the broad topography of the olivocerebellar system described previously (Groenewegen and Voogd, 1977; Azizi and Woodward, 1987; Buisseret-Delmas and Angaut, 1993; Voogd, 1995). The number of labeled climbing fibers in each rat ranged from 16 to 161 (mean $=53, \mathrm{SD}=41)$. Labeled climbing fibers clustered in a few longitudinal segments, each of which ranged from a part of a lobule to some consecutive lobules in length, and from 0.2 to $0.5 \mathrm{~mm}$ in width, indicating that such basic organization of the olivocerebellar projection as shown in Figures $3 b$ and $4 b$ is consistent throughout the cerebellar cortex and represents the basic organization of the olivocerebellar projection.

\section{Neighboring cells but not branches of a single axon may project to different bands}

Investigation of the arrangement of segments in the above 30 cases showed three distinct projection patterns: labeled climbing fibers could be clustered within (1) a single segment ( $n=8$ of 30) (Fig. 1b), (2) multiple separate segments in a single longitudinal band ( $n=17$ of 30 ) (Fig. 2), or (3) multiple segments in separate longitudinal bands located at different distances from the midline ( $n=5$ of 30 ). The relationship was examined between projection areas of a single axon and the multiple projection segments in the above cases ( 2 and 3 ). Cortical segments that were separate in the rostrocaudal direction but within a single longitudinal band were often innervated by a single neuron, as shown in Figure 2 [11 of 14 axons reconstructed in five rats in the above case (2)]. These 11 axons terminated in two segments that were separated rostrocaudally but contained all labeled climbing fibers in these five rats. 


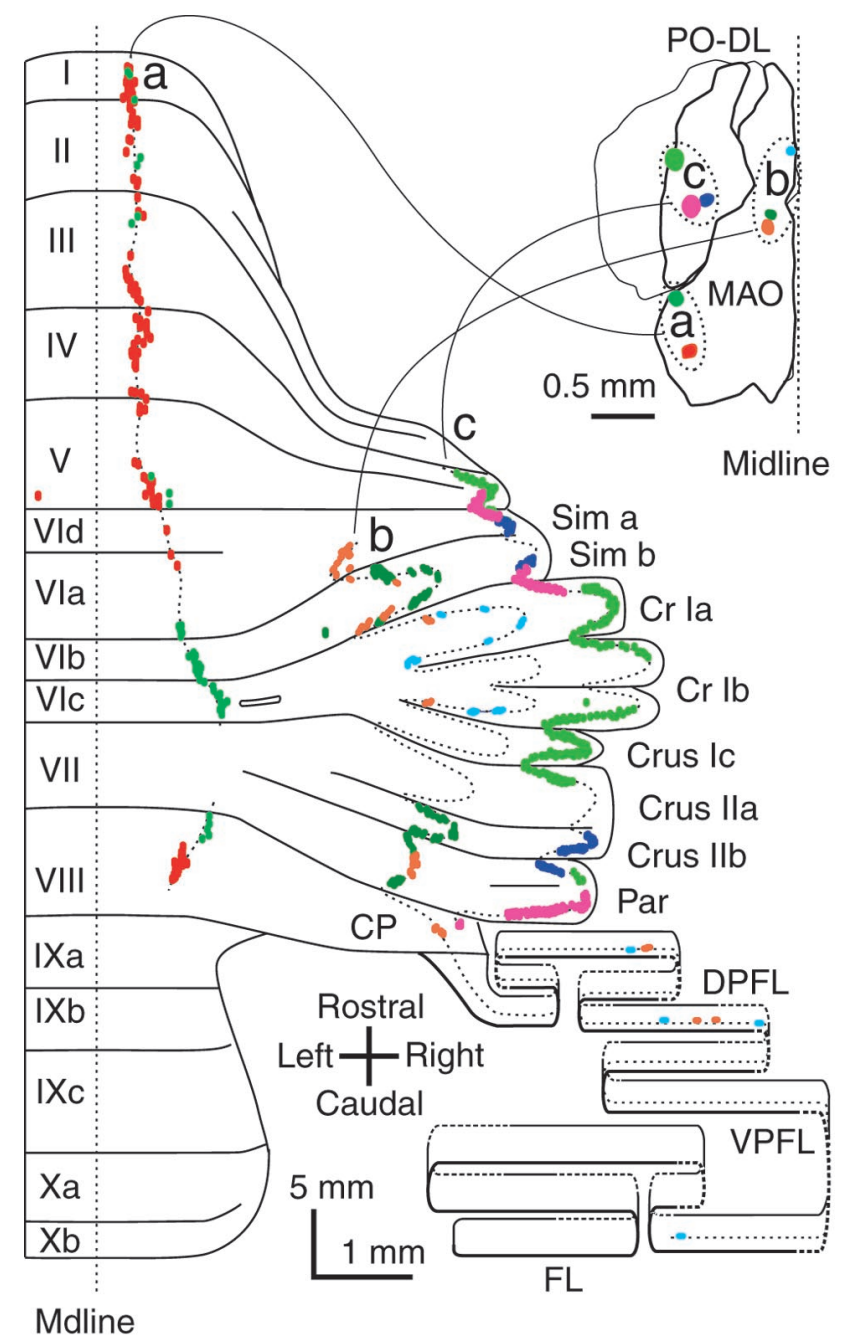

Figure 5. Projection patterns of climbing fibers originating from adjacent areas in the inferior olive showing complementary segments within the same longitudinal band. Climbing fibers labeled by small injections in two adjacent sites in the lateral and caudal portions of the MAO (green and red) $(a)$, three sites in the medial and rostral portions of the MAO (orange, dark green, and light blue) (b), and three sites in the lateral portion of the dorsal lamella of the principal olive (PO-DL) ( yellow-green, pink, and blue) $c$, Results of eight experiments are presented in three groups based on olivary injection sites and their related cerebellar projections ( $a$, vermis; $b$, medial hemisphere or pars intermedia; $c$, lateral hemisphere). Inset shows the dorsal view of the injection sites (colored spots) in the inferior olive. Dotted lines drawn along labeled climbing fibers in the cerebellar cortex indicate putative single longitudinal bands. Each colored $d o t$, which is often fused to others, indicates a labeled climbing fiber.

This indicated that a specific combination of rostrocaudally separate segments was coupled by axon collaterals of single olivocerebellar neurons, and these segments might be functionally interrelated. On the other hand, cortical segments that were separate mediolaterally were not simultaneously innervated by single olivocerebellar axons, suggesting that different populations of adjacent olivary neurons might be labeled by chance.

Only few climbing fibers were seen in the vermis and pars intermedia ipsilateral to the injection site in the inferior olive in 3 of the above 30 rats. These ipsilateral climbing fibers were located symmetrical to the midline with the contralateral main climbing fibers (Fig. 5a, red). The ipsilateral climbing fibers are formed by branches of olivocerebellar axons crossing the midline in the cerebellum, and the majorities of climbing fibers belonging to the same axons are located in the contralateral cerebellum (Sugihara et al., 1999). We regarded such ipsilateral projections as exceptional and did not include them in the above analysis.

\section{Complementary projection patterns of adjacent regions within single inferior olivary subnuclei produce longitudinal bands in the cerebellar cortex}

To understand the finer organization of individual longitudinal bands, small injections of tracer were made into adjacent parts of a specific olivary subnucleus (Fig. 5). Injections were made into the caudal MAO nucleus ( $n=2$ animals), the rostral MAO nucleus ( $n=3$ animals), and into the lateral portions in the dorsal lamella of the principal olive nucleus ( $n=3$ animals). Climbing fibers originating from neurons in the caudal MAO, rostral $\mathrm{MAO}$, and the principal olive projected to the vermis, the pars intermedia, and the hemisphere, respectively (Fig. 5a-c, three dotted longitudinal lines). These relationships between the subnuclei of the inferior olive and the locations of the bands in the cerebellar cortex were consistent with the gross topographical relationships of the olivocerebellar projection $(\mathrm{A}, \mathrm{C} 2$, and D1 zones) (Groenewegen and Voogd, 1977; Azizi and Woodward, 1987; Buisseret-Delmas and Angaut, 1993; Voogd, 1995), respectively. In the two cases of the projection to the vermis, climbing fibers were mostly distributed in small longitudinal segments in lobules I to V and apical lobule VIII in one case (red) and in lobules VIb-c and rostral lobule VIII in the other (green). These projection segments were arranged in the same longitudinal band in a roughly complementary manner. Similarly, in the other cases, climbing fiber distributions were roughly complementary and formed longitudinal bands (orange, dark green, and light blue in the pars intermedia, and yellow-green, pink, and blue in the hemisphere) (Fig. 5). These results indicated that slightly separate groups of olivary neurons in a single subnucleus projected to distinct sets of segments in the same longitudinal band in a complementary manner.

To examine further the organization of substructures and suprastructures of single longitudinal bands in the olivocerebellar projection, different volumes of tracer were injected to vary the sizes of labeling sites in the inferior olive, and the projection patterns of labeled climbing fibers were compared with each other (Fig. 6). The caudal portion of the MAO was used in this analysis, because it is relatively separated from the other subnuclei, and the probability of tracer spread into other subnuclei was low. With a small injection site (diameter, $0.1 \mathrm{~mm}$ ), there was a single longitudinal band with two small projection segments occupying lobules I-IV and VIII, and two of three reconstructed olivary axons innervated both segments by their ramification (Fig. 6a). With a slightly larger injection site (diameter, $0.3 \mathrm{~mm}$ ), the projection areas in the single longitudinal band extended in the rostrocaudal direction, covering most of the lobules except for some portions of lobules VIc, VII, IXa-b, and Xa-b with only slight extension in the lateral direction (Fig. 6b). A much larger injection site (diameter, $0.8 \mathrm{~mm}$ ) produced two additional longitudinal bands in the pars intermedia, but the original band near the midline now extended further in the rostrocaudal direction to cover nearly all of the lobules in the vermis without significant lateral extension (Fig. 6c). Similar changes in the projection patterns in relation to the sizes of the injection sites were observed in four other rats. All bands except those in the flocculus in Figure $6 c$ belonged to the so-called A zone that is innervated by the caudal portion of the MAO (Groenewegen and Voogd, 1977; Azizi and Woodward, 
a

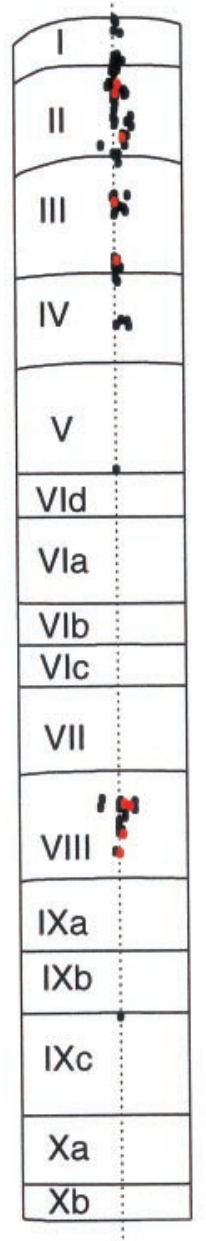

Midline b

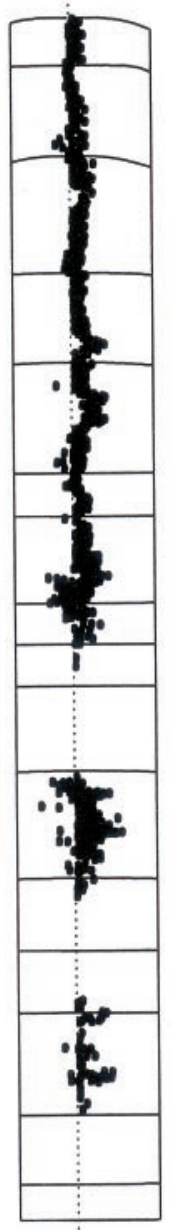

Midline
C

Figure 6. The distribution patterns of climbing fibers labeled by injections of different volumes of tracer into the inferior olive indicating substructures in the zonal organization of the olivocerebellar projection. $a$, Two longitudinal segments in lobules I-IV and VIII in the vermis along the midline produced by a small injection in the caudal portion of the MAO (right inset). Red dots indicate climbing fibers of a single reconstructed olivocerebellar axon terminating in both areas. $b$, Longitudinal band-shaped pattern occupying most of the lobules in the vermis along the midline produced by a medium-sized injection including the site in $a$. $c$, Striped pattern produced by an injection larger than in $b$ and covering the sites in $a$ and $b$ in the MAO. Curvatures of some lateral bands are attributable to the tilt of the longitudinal plane and the foliation of the cortex. Each dot, which is often fused to others, indicates a labeled climbing fiber.

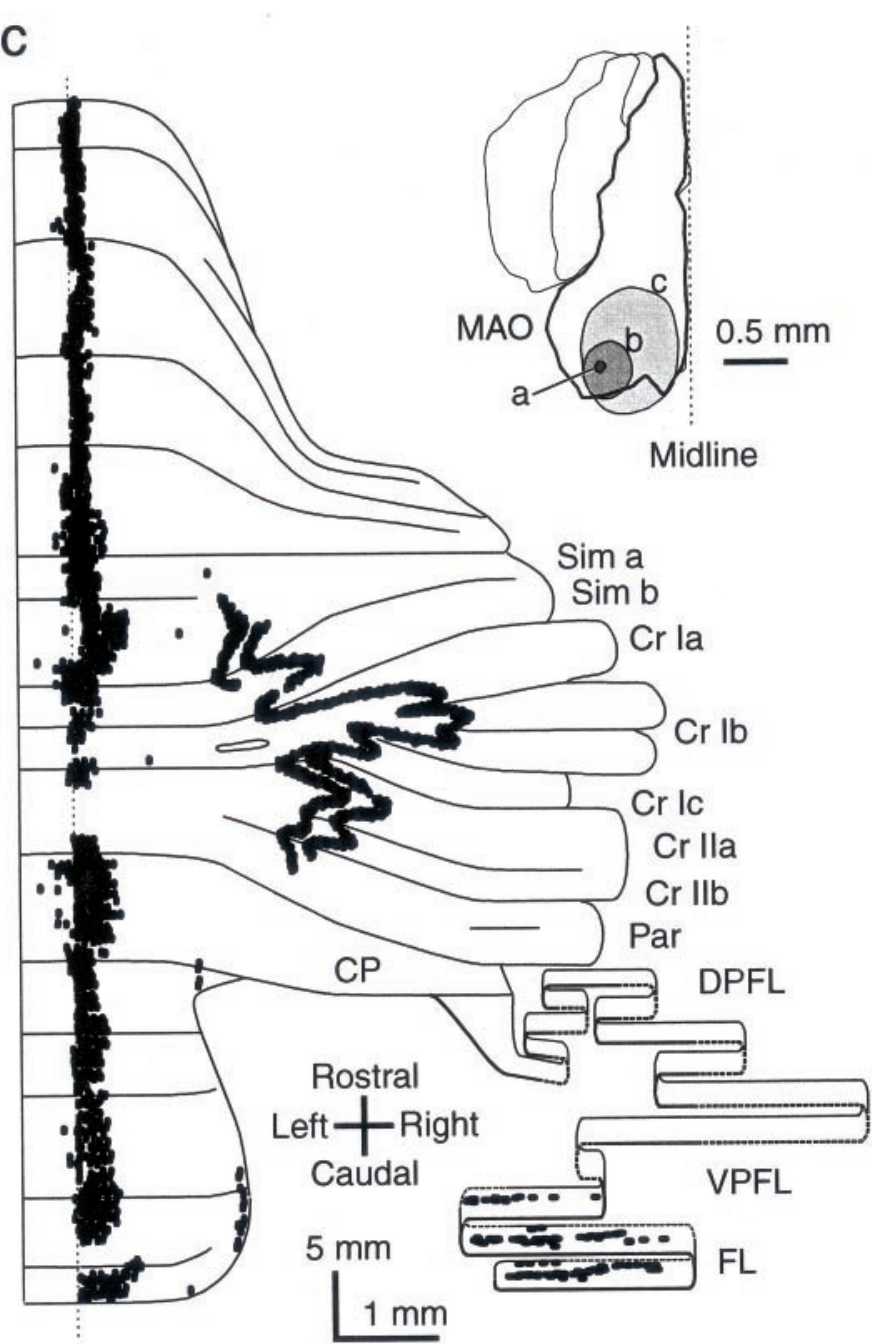

Midline
1987; Buisseret-Delmas and Angaut, 1993; Voogd, 1995). These results support the above conclusion that adjacent olivary neurons project to the same set of segments that are all arranged in a single longitudinal band, and slightly distant neurons project to different sets of segments in the same longitudinal band. Furthermore, more widely separated neurons project to different longitudinal bands. These findings on the substructure of the A-zone also suggest that individual cerebellar zones, which are defined according to the topographical innervation of the cortex by entire olivary subnuclei (Groenewegen and Voogd, 1977; Azizi and Woodward, 1987; Buisseret-Delmas and Angaut, 1993; Voogd, 1995), consist of several of the longitudinal bands.

\section{DISCUSSION}

The present study revealed that the traditionally defined anatomical zones defined on the basis of projections from the olivary subnuclei to the cerebellum have a substructure consisting of $0.2-$ to 0.3 -mm-wide longitudinal bands. These bands may provide a morphological substrate for physiologically defined narrow longitudinal zones (Oscarsson and Sjölund, 1977; Ito, 1984; Llinás and Sasaki, 1989; Ekerot et al., 1991; Jörntell et al., 2000; Hansen et al., 2000; Fukuda et al., 2001). The width of the band observed in the present morphological study is in accordance with the width determined by high synchrony of climbing fiber responses of multiple Purkinje cells (250-500 $\mu \mathrm{m}$; Fukuda et al., 2001). Furthermore, the present finding that adjacent olivary neurons project to the same band may explain the physiologically observed high-synchrony band (Fukuda et al., 2001), assuming that electrotonic coupling within nearby olivary neurons underlies the synchrony. However, physiological studies so far have not much clarified substructures of the longitudinal band. In the present morphological study, each longitudinal band was not evenly organized along its length, but consisted of several sets of segments that were arranged in a complementary manner within the overall confines of the band. We suggest that each segment represents the morphological substructure for the functional unit of the olivocerebellar projection. It is not clear whether neighboring segments may have clear borders or instead can be intermingled with each other at their border. The organization of such segments followed more or less the lobular division of the cerebellar cortex.

The segmental organization, revealed here, significantly increases the number of compartments in the cerebellar cortex in terms of the olivocerebellar projection. A very fine and complex topography may underlie the innervation of each segment by a small group of olivary neurons. The present labeling of a very localized population of olivary neurons has made it possible to reveal this fine topography. It has been hypothesized that origin- 
target matching with specific molecules underlies the formation of the topographical olivocerebellar projection (Chédotal et al., 1996). The fine topography revealed in this study suggests a sophisticated matching mechanism involving several of such molecules. Although mediolateral borders of olivocerebellar bands may coincide with the borders of molecular marker labelings (Wassef et al., 1992; Zagrebelsky et al., 1996), it is a remaining question whether molecular marker labelings (Hawkes et al., 1985) have any rostrocaudal organization compatible with the segmentation of each longitudinal band. Branches of a single olivocerebellar axon projecting to the same segments were sometimes completely separated in the deep cerebellar white matter (Figs. 1b, 3a, 4a), suggesting independent growth of these branches affected by the same target specificity.

Each small segment where adjacent olivary neurons project may be related to specific activity of climbing fiber input to the cerebellar cortex. Some segments reported here coincide well with physiologically defined zones in terms of specific receptive fields. For example, the segment in lobule I-IV in Figure 5 roughly coincides with the "a1 zone", which is responsive to somatosensory inputs from the proximal area in the rat (Jörntell et al., 2000). Lobules VIa-c and VII, in which the segments shown in Figure 3 are located, are related to eye movements (Godschalk et al., 1994). Some single olivary neurons innervated separate segments in the same band. This finding provides clear morphological evidence of interlobular branching of single olivocerebellar axons previously demonstrated electrophysiologically or by retrograde labeling studies (Armstrong et al., 1973; Brodal et al., 1980; Rosina and Provini, 1987; Apps, 2000). There seems to be some general principle organizing the location of the segments innervated by common axons across lobules. For example, individual axons often innervated segments in simple lobule and crus II-paramedian lobule (Fig. 2b,c; some in Fig. 5), or segments in lobules I-V and lobule V III (Fig. $2 a$; some in Fig. 5). Simple lobule and crus II-paramedian lobule may be functionally related to some extent, because both areas receive trigeminal input via mossy fibers in rats (Welker, 1987). Although little is known about the relationship of activities in lobules I-V and lobule VIII, the present results suggest that one would be found. None of the single olivary neurons thus far examined projected to segments located in mediolaterally separated longitudinal bands. This finding does not fit the electrophysiological finding that axon collaterals of single olivocerebellar axons often project to transversely separate microzones in cats (Ekerot and Larson, 1982). More detailed information on the organization of the corticonuclear and olivonuclear projections will be required to further understand the functional roles of the cerebellar compartmentalization in control of movement.

\section{REFERENCES}

Apps R (1998) Input-output connections of the "Hindlimb" region of the inferior olive in cats. J Comp Neurol 399:513-529.

Apps R (2000) Rostrocaudal branching within the climbing fibre projection to forelimb-receiving areas of the cerebellar cortical $\mathrm{Cl}$ zone. J Comp Neurol 419:193-204.

Armstrong DM, Harvey RJ, Schild RF (1973) The spatial organization of climbing fibre branching in the cat cerebellum. Exp Brain Res $18: 40-58$.

Azizi SA, Woodward DJ (1987) Inferior olivary nuclear complex of the rat: morphology and comments on the principles of organization within the olivocerebellar system. J Comp Neurol 263:467-484.

Brodal A, Walberg F, Berkley KJ, Pelt A (1980) Anatomical demonstra- tion of branching olivocerebellar fibres by means of a double retrograde labelling technique. Neuroscience 5:2193-2202.

Buisseret-Delmas C, Angaut P (1993) The cerebellar olivocorticonuclear connections in the rat. Prog Neurobiol 40:63-87.

Chédotal A, Pourquié O, Ezan F, Clemente HS, Sotelo C (1996) BEN as a presumptive target recognition molecule during the development of the olivocerebellar system. J Neurosci 16:3296-3310.

Eccles JC, Ito M, Szentágothai J (1967) The cerebellum as a neuronal machine. New York: Springer.

Ekerot C-F, Larson B (1982) Branching of olivary axons to innervate pairs of sagittal zones in the cerebellar anterior lobe in the cat. Exp Brain Res 48:185-198.

Ekerot C-F, Grawicz M, Schouenborg J (1991) Topography and nociceptive receptive fields of climbing fibres projecting to the cerebellar anterior lobe in the cat. J Physiol (Lond) 441:257-274.

Fukuda M, Yamamoto T, Llinás R (2001) The isochronic band hypothesis and climbing fibre regulation of motricity: an experimental study. Eur J Neurosci 13:315-326.

Godschalk M, Van der Burg J, Van Duin B, De Zeeuw CI (1994) Topography of saccadic eye movements evoked by microstimulation in rabbit cerebellar vermis. J Physiol (Lond) 480:147-153.

Groenewegen HJ, Voogd J (1977) The parasagittal zonation within the olivocerebellar projection. I. Climbing fiber distribution in the vermis of the cat cerebellum. J Comp Neurol 174:417-488.

Hansen CL, Chen G, Ebner TJ (2000) Role of climbing fibers in determining the spatial patterns of activation in the cerebellar cortex to peripheral stimulation: an optical imaging study. Neuroscience 96:317-331.

Hawkes R, Colonnier M, Leclerc N (1985) Monoclonal antibodies reveal sagittal banding in the rodent cerebellar cortex. Brain Res 333:359-365.

Ito M (1984) The cerebellum and neural control. New York: Raven.

Jörntell H, Ekerot C-F, Garwicz M, Luo X-L (2000) Functional organization of climbing fiber projection to the cerebellar anterior lobe of the rat. J Physiol (Lond) 522:297-309.

Llinás R, Sasaki K (1989) The functional organization of the olivocerebellar system as examined by multiple Purkinje cell recordings. Eur J Neurosci 1:587-602.

Llinás R, Baker R, Sotelo C (1974) Electrotonic coupling between neurons in cat inferior olive. J Neurophysiol 37:560-571.

McCurdy ML, Houk JC, Gibson AR (1998) Organization of ascending pathways to the forelimb area of the dorsal accessory olive in the cat. J Comp Neurol 392:115-133.

Oscarsson O, Sjölund B (1977) The ventral spino-olivocerebellar system in the cat. I. Identification of five paths and their termination in the cerebellar anterior lobe. Exp Brain Res 28:469-486.

Palay SL, Chan-Palay V (1974) Cerebellar cortex. Cytology and organization. New York: Springer.

Ramón y Cajal S (1911) Histologie du Système Nerveux de l'Homme et des Vertébrés, Vol II. Paris: Maloine.

Rosina A, Provini L (1987) Spatial distribution of axon collaterals of single inferior olive neurons. J Comp Neurol 256:317-328.

Ruigrok TJ, Osse RJ, Voogd J (1992) Organization of inferior olivary projections to the flocculus and ventral paraflocculus of the rat cerebellum. J Comp Neurol 316:129-150.

Schild RF (1970) On the inferior olive of the albino rat. J Comp Neurol 140:255-260.

Shinoda Y, Yokota J, Futami T (1981) Divergent projection of individual corticospinal axons to motoneurons of multiple muscles in the monkey. Neurosci Lett 23:7-12.

Sugihara I, Wu H-S, Shinoda Y (1999) Morphology of single olivocerebellar axons labeled with biotinylated dextran amine in the rat J Comp Neurol 414:131-148.

Tan J, Gerrits NM, Nanhoe R, Simpson JI, Voogd J (1995) Zonal organization of the climbing fiber projection to the flocculus and nodulus of the rabbit: a combined axonal tracing and acetylcholinesterase histochemical study. J Comp Neurol 356:23-50.

Voogd J (1995) Cerebellum. In: The rat nervous system, Ed 2. (Paxinos G, ed), pp 309-350. San Diego: Academic.

Wassef M, Angaut P, Arsenio-Nunes L, Bourrat F, Sotelo C (1992) Purkinje cell heterogeneneity: its role in organizing the topography of the cerebellar cortex connections. In: The cerebellum revisited (Llinás R, Sotelo C, ed), pp 5-21. New York: Springer.

Welker W (1987) Spatial organization of somatosensory projections to granule cell cerebellar cortex: Functional and connectional implications of fractured somatotopy (summary of Wisconsin studies). In: New concepts in cerebellar neurobiology (King, JS, ed), pp 239-280. New York: Liss.

Zagrebelsky M, Rossi F, Hawkes R, Strata P (1996) Topographically organized climbing fibre sprouting in the adult rat cerebellum. Eur J Neurosci 8:1051-1054. 